\title{
Perfil inmunohistoquímico del cáncer de mama en pacientes de un hospital general de Arequipa, Perú
}

\author{
Immunohistochemistry profile of breast cancer in a general hospital in Arequipa, Peru
}

Gonzalo Mendoza del Solar ${ }^{1,2, a, b}$, Anuska Echegaray ${ }^{1, c}$, Cesar Caso ${ }^{1, d}$

\section{RESUMEN}

Los avances logrados en biología molecular permiten personalizar el tratamiento del cáncer de mama, siendo las pruebas genéticas de difícil acceso para nuestra población, se utiliza el perfil inmunohistoquimico. Objetivos: Determinar el perfil inmunohistoquimico del cáncer de mama en pacientes atendidas en un hospital general de Arequipa, Perú. Material y métodos: Estudio descriptivo, retrospectivo, realizado en el Hospital Goyeneche de Arequipa, Perú, entre enero del 2007 y diciembre del 2012. Se incluyeron 65 mujeres con diagnóstico de cáncer de mama a quienes se les realizó estudio de receptores hormonales y Cerb-2 por inmunohistoquimica. Resultados: El 50,8\% de casos expresaron RE, el 44,6\% expresaron RP y el 23,1\% expresaron Cerb-2. El 30,8\% de mujeres con cáncer de mama fue triple negativo. Conclusiones: El perfil inmunohistoquimico de las pacientes con cáncer de mama es similar al de otros estudios. Más de la mitad, fueron receptores estrogénicos positivos por lo tanto sensibles a manipulación hormonal, un quinto de casos expresan Cerb-2, por ende candidatas a recibir tratamiento con anticuerpo monoclonal y un tercio fue triple negativo, por lo tanto de mal pronóstico.

PALABRAS CLAVE: Neoplasias de la mama, marcadores biológicos, inmunohistoquímica. (Fuente: DeCS BIREME).

\section{SUMMARY}

Immunohistochemistry results allow for a personalized treatment of patients with breast cancer. Objective: To determine the immunohistochemistry profile of patients with breast cancer in a general hospital in Arequipa, Peru. Methods: Retrospective study carried out at Hospital Goyeneche from January 2007 to December 2012. Hormonal receptor studies and Cerb-2 immunohistochemistry panels were performed. Results: 50,8\% of cases had RE, 44,6\% had RP and 23,1\% had Cerb-2; 30,8\% of breast cancers had all three negative markers. Conclusions: The immunohistochemistry profile of this setting is similar than reported elsewhere. More than half of patients had positive estrogenic receptors and were amenable of hormonal manipulation; one fifth of cases were Cerb-2 positive amenable of treatment with monoclonal antibodies and one third had negative markers are therefore poor prognosis.

KEYWORDS: Breast neoplasms, biological markers, immunohistochemistry. (Source: MesH NLM).

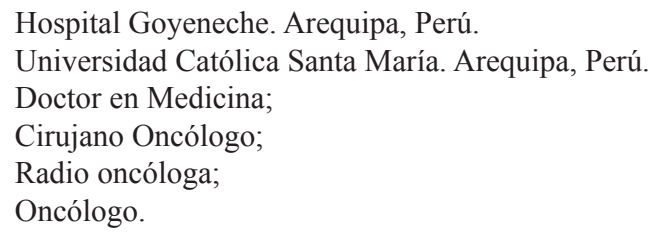




\section{INTRODUCCIÓN}

El cáncer de mama es el cáncer que afecta a mujeres con más frecuencia a nivel mundial. Según la última publicación de la Agencia Internacional de Investigación en Cáncer, la incidencia mundial en el 2012 fue 1677000 casos, representando el 25\% de todos los cánceres diagnosticados en el año. La incidencia mundial del cáncer de mama es de 38,9 casos por cada cien mil mujeres (1). En el Perú, el cáncer de mama es la neoplasia más frecuente en mujeres con una incidencia de 35,7 casos por cada 100 000 (2) y en Arequipa ocupa el segundo lugar, después del cáncer de cuello uterino, con una incidencia de 26,1 casos por cada 100000 mujeres (3).

A pesar que en los últimos años la incidencia de cáncer de mama se ha incrementado en forma constante, la mortalidad ha empezado a disminuir (4). Esto debido a dos hechos importantes, el aumento de despistaje oportuno y los avances en el tratamiento del cáncer de mama, tanto en las terapias locales, cirugía y radioterapia, como en las terapias sistémicas con el uso de neo-adyuvantes y adyuvantes con quimioterapia, hormonoterapia y agentes biológicos contra blancos terapéuticos específicos. La biología molecular nos ha enseñado la existencia de varios subtipos de cáncer de mama, ahora bien tipificados con el uso de pruebas genéticas (5), que permiten pronosticar la respuesta a diferentes tratamientos y la sobrevida, constituyéndose en importante arma terapéutica en el momento de seleccionar la terapia a instaurarse (6).

Las pruebas genéticas son costosas y no están al alcance de todos los pacientes, sin embargo con pruebas de inmunohistoquimica se puede lograr diseñar tratamientos de acuerdo a los subtipos del cáncer de mama, en concordancia con guías de terapia de consenso como las de The European Society for Medical Oncology (ESMO), American Society of Clinical Oncology (ASCO), The National Institute for Health and Care Excellence (NICE) y The National
Comprehensive Cancer Network (NCCN) (7), esta última es la que venimos utilizando en el Departamento de Oncología del hospital.

El objetivo del trabajo fue determinar el perfil de receptores moleculares del cáncer de mama de pacientes tratadas en un hospital general de Arequipa, Perú.

\section{MATERIAL Y MÉTODOS}

Estudio retrospectivo, realizado entre el 1 de enero del 2007 y el 31 de diciembre del 2012 en el Departamento de Oncología del Hospital Goyeneche. Este hospital pertenece a la Gerencia Regional de Salud de Arequipa y es el primer centro oncológico de la Región congregando a oncólogos quirúrgicos, médicos y radio-oncólogos.

Se incluyeron en el estudio, todas las pacientes con cáncer de mama a cuyas biopsias o piezas operatorias se les había realizado estudios inmunohistoquímicos para determinar el estado de los receptores estrogénicos, de progesterona y Cerb-2. Se encontraron 65 pacientes que cumplían los criterios de inclusión

Se revisaron las historias clínicas y se registraron los datos en una ficha.

Se utilizó estadística descriptiva, se determinaron frecuencias absolutas y relativas.

\section{RESULTADOS}

La expresión de los receptores de estrógenos, progesterona y para Cerb-2, se muestran en la tabla 1. El 50,8\% expresaron receptores para estrógenos.

Veinte $(30,8 \%)$ mujeres con cáncer de mama no tuvieron expresión de alguno de los receptores, es decir fueron triple negativo.

Tabla 1. Expresión de receptores según inmunohistoquimica en pacientes con cáncer de mama. Arequipa, Perú.

\begin{tabular}{lllll}
\hline & \multicolumn{2}{c}{ POSITIVO } & \multicolumn{2}{c}{ NEGATIVO } \\
\hline & $\mathbf{n}$ & $\mathbf{\%}$ & $\mathbf{n}$ & $\mathbf{\%}$ \\
Receptor de Estrógeno & 33 & 50,8 & 32 & 49,2 \\
Receptores de Progesterona & 29 & 44,6 & 36 & 55,4 \\
Receptores Cerb-2 & 15 & 23,1 & 50 & 76,9 \\
\hline
\end{tabular}




\section{DISCUSIÓN}

La expresión de receptores de estrógeno se encontró en $50,8 \%$; cifra similar al encontrado en un estudio realizado en Cuba (8), donde encontraron receptores hormonales positivos en 53\% de casos, y en México (9). Sin embargo, en Colombia hallaron $88 \%$ de expresión de este receptor (10). La determinación de la expresión de este receptor hormonal es importante porque la terapia hormonal solo deberá ser indicada a las pacientes que expresen RE positivos (11), no como anteriormente se hacía de indicar tamoxifeno a todas las pacientes luego de la cirugía y quimioterapia.

A pesar de ser un importante factor predictivo de respuesta al tratamiento hormonal, al parecer no lo es de supervivencia. En un estudio se encontró que 5 años después del diagnóstico, las mujeres con un tumor receptor de estrógeno negativo tienen el mismo riesgo de morir como consecuencia del cáncer de mama que las mujeres con un tumor RE positivo (12).

La frecuencia de receptor de progesterona positivo se encontró en $44,6 \%$ de los casos, similar al encontrado en Cuba 49\% (8) y en España en 57\% (13) Este resultado también es de importancia ya que la presencia de ambos receptores hormonales en un mismo tumor aumenta la probabilidad de respuesta al tratamiento hormonal desde $60 \%$ con receptor de estrógeno positivo, a $75-80 \%$ si ambos están presentes (14).

La expresión del oncogén Cerb - B2, se encontró en $23,1 \%$; éste resultado concuerda con otro estudio realizado en México donde se halló 20,5\% de casos Cerb-B2 positivo (9), $21 \%$ en el Hospital Nacional Edgardo Rebagliati Martins de EsSalud (15) y 21,4\% en España (13). Sin embargo, estudios realizados en Costa Rica y Colombia encuentran frecuencias menores, 7,7 y $1,16 \%$ de casos respectivamente $(10,16)$. La expresión de este oncogén se considera desfavorable para la evolución de la enfermedad, puesto que, se asocia con mayor infiltración del tumor y peor pronóstico (17).

En veinte $(30,8 \%)$, la expresión fue negativa a los tres receptores (triple negativo), de esto podemos inferir que casi la tercera parte de casos de cáncer de mama han tenido mayor agresividad y peor sobrevida. Este dato es similar al encontrado en México, donde se halló 28,6\% (9). En otros estudios sin embargo, se encuentra cifras menores entre 12-17\% (18), en Costa Rica reportan subtipo triple negativo en $18 \%$ (16). Esta variación en las cifras podría deberse a las técnicas de fijación del tejido que se utilizan en los diversos países para realizar el estudio inmunohistoquimico (19).

El tamaño de la muestra y la falta de estandarización de los laboratorios que realizan las pruebas de inmunohistoquimica son importantes limitaciones de este estudio.

El perfil inmunohistoquimico de las pacientes con cáncer de mama, atendidas en el Departamento de Oncología del Hospital Goyeneche, es similar a los descritos en otras ciudades de Ibero América.

\section{Declaración de Financiamiento y de Conflictos de Intereses:}

Los autores declaran que el financiamiento del estudio ha sido con recursos propios y expresan no tener conflictos de interés alguno.

\section{Contribución de autoría:}

GMdS, AE y CC participaron en el diseño del estudio, recolección de los datos, interpretación de los resultados y redacción del manuscrito.

\section{Correspondencia:}

Gonzalo Mendoza del Solar

Correo electrónico: gonzalo@mendozadelsolar.com

\section{REFERENCIAS BIBLIOGRÁFICAS}

1. International Agency for Research on Cancer, World Health Organization. GLOBOCAN 2012: Estimated cancer indicence in prevelence worldwide in 2012. Lyon: IARC. (Citado el 2 de febrero del 2014) Disponible en: http://globocan.iarc.fr/Pages/fact_ sheets_cancer.aspx

2. Ministerio de Salud. Norma Técnico oncológica para la prevención, diagnostico y tratamiento temprano del cáncer de mama a nivel Nacional.- Lima: MINSA: 2008.

3. Ministerio de Salud. Registro de Cáncer Poblacional de Arequipa, Perú. Arequipa: Instituto Regional de Enfermedades Neoplásicas del Sur; 2011.

4. American Cancer Society. Cancer facts \& figures 2009. Atlanta, Ga: American Cancer Society; 2009.

5. Henry NL, Hayes DF. Use of gene-expression profiling to recommend adjuvant chemotherapy for breast cancer. Oncology (Williston Park). 2007; 21:1301-1309.

6. Albain KS, Paik S, van't Veer L. Prediction of 
adjuvant chemotherapy benefit in endocrine responsive, early breast cancer using multigene assays. Breast. 2009; 18(S3):S141-S145.

7. Clinical practice guidelines in oncology: breast cancer. Pennsylvania: National Comprehensive Cancer Network (NCCN). (Citado el 25 de noviembre del 2013). Disponible en: http://www. nccn.org/professionals/physician_gls/PDF/breast.pdf

8. Álvarez R, Escobar X, Camacho R, et al. Receptores hormonales y otros factores pronósticos en cáncer de mama en Cuba. Cancerología. 2008; 3: 19-27.

9. Muñoz-Duran L, Álvarez-Mondaca J, EspinoVillalobos J, Murillo-Llanes J, Alejo-Armenta LN. Receptores de estrógeno, progesterona y Her 2/ Neu, en pacientes con cáncer de mama tratadas en el centro estatal de oncología de Sinaloa. A S Sin. 2008; 2(4):126-131.

10. Silvera L, Caez C, Camargo P, et al. Análisis de los factores inmunohistopatológicos (receptores hormonales, estrógenos, progesterona y ERB2) asociados al pronóstico del cáncer de mama en la población de Barranquilla (2004- 2005). Salud Uninorte 2007; 23 (2): 150 -161.

11. Sotiriou C, Lajos Pusztai L. Gene-expression signatures in breast cancer. N Engl J Med 2009; 360:790-800.

12. Colzani E, Liljegren A, Johansson A, et al. Prognosis of patients with breast cancer: causes of death and effects of time since diagnosis, age, and tumor characteristics. JCO. 2011; 29(30):4014-4021.
13. Cabrero A, Picón G, Gisela K, Valencia R, Barroso S. Cáncer de mama metastásico: estudio clínicopatológico de 300 casos. Patología. 2010; 48(1): 1822.

14. Copeland EM. La mama: manejo multidisciplinario de las enfermedades benignas y malignas. Buenos Aires: Editorial Médica Panamericana; 1993. p. 438.

15. Pareja Zea, Ana Sofía. Características epidemiológicas y biomarcadores por inmunohistoquimica en mujeres con cáncer de mama en el Hospital Nacional Edgardo Rebagliati Martins Lima 2007. Universidad Católica de Santa María Arequipa, Perú.

16. Quirós-Alpízar J, Jiménez-Rodríguez Y, JiménezMontero E, Monge-Víquez M, Salazar-Sánchez L. Carcinomas invasores triples negativos de la glándula mamaria: incidencia y características clínicopatológicas. Acta Med Costarricense. 2010; 52(2): 90-95.

17. Arafah M. Correlation of hormone receptors with Her-2 Neu protein expression and the histological grade in invasive breast cancers in a cohort of Saudi Arabia. Turk Patoloji Derg. 2012; 28(1):38-43.

18. Foulkes W, Smith I, Reis-Filho J. Triple-negative breast cancer. N Engl J Med. 2010; 363:1938-48.

19. Oyama T, Ishikawa Y, Hayashi M, Arihiro K. The effects of fixation, processing and evaluation criteria on immunohistochemical detection of hormone receptors in breast cancer. JCO. 2007; 14(2):182-188.

Recibido: 23/04/2014

Aceptado: 23/12/2014 